\title{
Integration of genomics into the electronic health record: mapping terra incognita
}

\author{
Joseph L. Kannry, MD'1 and Marc S. Williams, MD²
}

The mapping of the human genome has enabled new exploration of how genetic variations contribute to health and disease. This research has been very successful not only in shedding light on how genetic variants influence susceptibility to common, chronic diseases but also in playing an instrumental role in the discovery of new biologic pathways and drug targets. Despite the growing body of literature of the value of pharmacogenomic variants to drug efficacy and safety and disease variants for risk, significant challenges remain in translating this information into clinical practice at the point of care, let alone integrating it into electronic health records (EHRs).

The adoption rate of EHRs for clinical care has accelerated because of the Center for Medicare and Medicaid Services' EHR Incentive Program, which incentivizes providers and hospitals for meaningfully using EHRs. ${ }^{1,2}$ This program, also known as the Meaningful Use program, has reimbursed physicians and hospitals a total of $\$ 14.3$ billion as of 30 April 2013. ${ }^{3}$ To date, no commercial EHR system has been described that systematically integrates genetic or genomic data, let alone uses this information to translate disease risk into treatment recommendations. Even pharmacogenetic information, one of the emerging targets for use of genomic information in health care, is rarely utilized to reduce adverse drug events and improve drug efficacy, despite increasing evidence of clinical validity and utility. ${ }^{4-7}$ Use of genetic testing for commonly used medications such as warfarin and clopidogrel can improve initial dosing, ${ }^{8}$ and there is early evidence that this can reduce recurrent myocardial infarction, stent thrombosis, and death. ${ }^{9}$

Despite the reluctance shown by clinicians, many academic centers are planning for or launching start-up efforts in genomic exploration that depend on EHR integration for their success. The National Human Genome Research Institute-sponsored Electronic Medical Records and Genomics (eMERGE) Network is a federally funded consortium of nine institutions with unique and valuable pioneer experience using a variety of commercial and homegrown EHRs. This special issue will use the experience of the eMERGE sites to illustrate challenges and solutions for integrating genomic data into the EHR, creation of integrated genomic decision support, and the human and electronic processes, including standards required for such successful integration. The special issue is intended to provide guidance to the many sites that are embarking upon or planning for the integration and implementation of genomic medicine into clinical care. Authors of each article were asked to provide guidance in the form of a "how to" and "what to think about" for anyone tasked with launching a genomics program and integrating these data into the EHR at the point of care.

\section{SUMMARY OF PAPERS IN THE SPECIAL ISSUE}

The articles in this special issue for the most part are authored by representatives of the eMERGE consortium's EHR integration workgroup. To provide context for readers not familiar with eMERGE, the article by Gottesman et al. ${ }^{10}$ provides a description of the consortium and its current working groups. It summarizes work completed in phase 1 of funding and details ongoing activities in phase 2. Phase 2 is of particular relevance to the topic of the special issue because increased emphasis on implementation of genomic medicine into clinical activities has necessitated more complete exploration of the capability of current EHR systems for genomic medicine (for interested readers additional articles on the eMERGE network are available). ${ }^{11-13}$

Successfully integrating genomics into clinical care requires a vision, a strategy that will achieve the vision, and an actionable implementation plan. A key to successful implementation is engagement with key stakeholders. Hartzler et al. ${ }^{14}$ articulate the need for a broad range of stakeholders, including providers, ancillary personnel, administrators, information technology staff, institutional leadership, and patients, if genomic medicine implementation is to be successful. Their article describes different ways to meaningfully engage stakeholders to ensure that there is support for the vision. The second part of the article provides an implementation roadmap that relies on ongoing involvement with stakeholders using an iterative approach. 
This allows for "course corrections" based on stakeholder input throughout the process, greatly increasing the likelihood of success. Significant tasks include:

1. A needs assessment,

2. Development of use cases,

3. Design and testing of the proposed tool (ideally in a developmental environment),

4. Deployment of the tool, and

5. Evaluation of the success of the implementation.

Although the definition of the EHR is straightforward, EHRs vary dramatically in their features, capabilities, and ease of use. Even the same type of EHR can differ significantly depending on the specifics of the implementation, leading some to remark "If you've seen one EHR implementation, you've seen one EHR implementation." In their article, Marsolo and Spooner ${ }^{15}$ identify five core EHR functions that are needed if genomic information is to be used in clinical care. These include:

1. Storing genetic information as structured data;

2. The structured data must be standards based to allow the information to move between different EHR systems;

3. Rich phenotypic information must also be stored as structured data and associated with relevant genetic information;

4. The data must be available for use by rules-based decision-support engines;

5. The EHR must be able to obtain and display information needed by the clinician to interpret the genotypic and phenotypic data.

Challenges exist in all of these areas, and the authors explore these as well as the impact the type of EHR and EHR implementation have on the desired functionality. Finally, the differences in handling research and clinical data are discussed.

The National Human Genome Research Institute has been actively funding research to explore the potential of wholegenome and whole-exome sequencing to generate new knowledge and improve patient outcomes through clinical research pilots. One such project is the Clinical Sequencing Exploratory Research (CSER) consortium. The CSER consortium, like the eMERGE consortium, is a U01-funded cooperative agreement that funds several independent projects that are exploring various applications of whole-genome and whole-exome sequencing; it convenes representatives from the various projects to work on cross-cutting issues that affect all projects. Because the projects are using EHRs to aid in the storage and use of sequencing data, a workgroup addressing EHR issues has also been created in the CSER consortium. Many, if not most, of the issues are also relevant to the eMERGE consortium, so each workgroup has a designated liaison to the other project's workgroup. The article by Tarczy-Hornoch et al. ${ }^{16}$ describes the results of a survey completed by CSER consortium members about their respective EHRs-all of which are from the same commercial vendor. The survey shows that even though all CSER projects are using the same sequencing technology and the same commercial EHR, there is a great diversity of approach for the return of results. Significant variation is noted in variant databases, variant predictors, allele frequency, variant curation strategies, laboratory information systems, and interfaces between the laboratory information systems and the EHR. Each site has a custom implementation that requires significant infrastructure and support.

Health-care delivery increasingly depends on the generation and analysis of data. Genomic medicine is no different. Data can be structured or unstructured. Structured data are able to be "read" by a computer and can be used to run clinical decisionsupport engines. ${ }^{17}$ Unfortunately, most data obtained in health care is unstructured. Kho et al. ${ }^{18}$ examine data elements relevant for genomic medicine that are currently captured in EHR systems and compare these data to genomic data. Demographic data (age, race, sex, and ethnicity) may affect the prior probability of disease, informing genetic risk predictions. Medication lists and orders can identify pharmacogenetic applications. Family history remains an important element that in many cases provides superior risk estimates as compared with genomic risk factors. Kho et al. note that genomic data will initially resemble genetic data, which currently represent a mixture of structured and unstructured data. Even if more structured data are able to be generated in clinical encounters, there remain many obstacles to their optimal use. Finally, issues related to privacy and confidentiality of genomic data are explored.

The article by $\mathrm{Ury}^{19}$ also addresses issues related to data but explores these from the EHR vendor perspective. Ury defines three data types: granular (structured), textual (unstructured), and image. Most data have a very short life span, with the need to access older data rarely required. Genomic data is different and will need to be maintained and accessed over the lifetime of the patient. Potential solutions for this problem will be discussed.

The amount of data needed to effectively care for patients already exceeds human cognitive capacity even before genomic data are added. This recognition has led to the development of functional EHRs with clinical decision-support (CDS) capabilities that can assist clinicians by retrieving and synthesizing data needed for a given clinical situation. This approach will be necessary if genomic medicine is to be successfully implemented. Overby et al. ${ }^{17}$ describe system desiderata needed to support stakeholder and informational needs. The differences between open or external CDS and internal CDS are examined with respect to updating, scalability, and handling of personal health information. At present, successful CDS solutions are developed in a single organization and are difficult to transfer to and implement in other organizations. Emerging evidence for the effectiveness of CDS in genomic medicine will be discussed.

One of the newest National Institutes of Health initiatives relates to projects involving "big data." Chute et al. ${ }^{20}$ discuss the opportunities that big data present to facilitate discoveries that were not possible even a few years ago. This will be of particular 
importance to medicine for a variety of reasons, not the least of which is the complexity of deriving phenotypes from clinical data. This is of particular importance for genomic medicine because developing genotype/phenotype correlations has proven to be challenging. Chute et al. note four opportunities for big data in health care:

1. Generation of new knowledge,

2. Dissemination of knowledge,

3. Translation of personalized medicine into clinical practice, and

4. Transforming health care by empowering patients with data.

Significant gaps exist in clinical genomics, including standards for coding and transmission of data. The article reviews these gaps and proposes solutions, including the role meaningful use could play in the development of solutions.

One of the first uses for genomic information in clinical care is related to genomic information that predicts response to drugs, i.e., pharmacogenomics. Peterson et al. ${ }^{21}$ describe the successful implementation of pharmacogenomic applications in the context of a homegrown EHR. Functionality developed for the application addressed problems that included storage of and access to pharmacogenomic data, interpretation in the appropriate clinical context, development of CDS, and presentation of results to clinicians and patients. Homegrown EHRs represent a good test bed for novel and innovative health information technologies but do not promote solutions that are sustainable and generalizable. Solving this problem will require iterative refinements that can promote rapid customization and implementation within different EHR systems.

It is clear that for genomic medicine to be successful, EHRs cannot simply be electronic representations of paper charts. However, functional implementation will raise issues of equitable access and privacy. The article by Hazin et al. ${ }^{22}$ explores issues related to ethical, legal, and social issues associated with genomics in the EHR. Genomic medicine has been proposed as a way to reduce health disparities, but if that vision is to be realized, patients will need to have equitable access to genetic testing, fully functional EHRs with genomic CDS, and knowledge repositories that can store and transmit the data to wherever the patient and provider are making decisions. Significant needs exist to improve genomic literacy for patients and providers. The role of educational materials accessible in EHRs and personal health records using novel delivery methods is explored. The need to share information relevant to the patient must be balanced with privacy and security. Patients share responsibility for privacy and security and have the most to gain from solutions that address storage, reinterpretation, and liability issues. Potential policy solutions are discussed.

The nature of the topic introduces a lexicon that may be unfamiliar to many readers of Genetics in Medicine. In recognition of this problem, a glossary of terms used in the articles has been included as a reference for the reader (page 846).
EHRs represent a necessary component to the realization of genomic medicine, and integration with EHRs is a primary focus of stage 2 of eMERGE. The articles in this special issue of Genetics in Medicine should assist system organizational leaders, both operational and academic, who are charged with implementing genomic medicine to leverage the EHR for both data and clinical integration. The articles draw upon the extensive experience of the eMERGE network informatics members and are meant to provide guidance based on important lessons learned by the eMERGE consortium. It is hoped that the issue will serve as a useful reference and guide over the next few years until the integration into and use of genomics data in the EHR becomes a routine part of clinical care.

\section{ACKNOWLEDGMENTS}

M.S.W. has funding from the National Human Genome Research Institute eMERGE project Geisinger E-Genomic Medicine Program (NHGRI grant no. 1U01HG006382-01) and the eMERGE-PGX supplement 1 (NIH grant no. 3U01HG006382-02S1). J.M.K. has funding from the National Human Genome Research Institute eMERGE project (grant no. U01HG006380).

\section{DISCLOSURE}

The authors declare no conflict of interest.

\section{REFERENCES}

1. Medicare and Medicaid programs; electronic health record incentive program. Final rule. Federal Register 2010;75(144):44313-44588.

2. Medicare and Medicaid programs; electronic health record incentive programstage 2. Final rule. Federal Register 2012;77(171):53967-54162.

3. CMS. EHR Incentive Program: Data and Reports. http://www.cms. gov/Regulations-and-Guidance/Legislation/EHRIncentivePrograms/ DataAndReports.html Accessed 27 July 2012

4. Mrazek DA, Lerman C. Facilitating clinical implementation of pharmacogenomics. JAMA 2011;306:304-305.

5. Crews KR, Hicks JK, Pui CH, Relling MV, Evans WE. Pharmacogenomics and individualized medicine: translating science into practice. Clin Pharmacol Ther 2012;92:467-475.

6. Roden DM, Xu H, Denny JC, Wilke RA. Electronic medical records as a tool in clinical pharmacology: opportunities and challenges. Clin Pharmacol Ther 2012;91:1083-1086.

7. Kushniruk AW, Borycki EM, Kannry J. Commercial versus in-situ usability testing of healthcare information systems: towards "public" usability testing in healthcare organizations. Stud Health Technol Inform 2013;183:157-161.

8. Johnson JA, Gong L, Whirl-Carrillo M, et al.; Clinical Pharmacogenetics Implementation Consortium. Clinical Pharmacogenetics Implementation Consortium Guidelines for CYP2C9 and VKORC1 genotypes and warfarin dosing. Clin Pharmacol Ther 2011;90:625-629.

9. Scott SA, Sangkuhl K, Stein CM, et al. Clinical Pharmacogenetics Implementation Consortium (CPIC) guidelines for cytochrome P450-2C19 (CYP2C19) genotype and clopidogrel therapy: 2013 Update. Clin Pharmacol Ther 2013; e-pub ahead of print 22 May 2013.

10. Gottesman O, Kuivaniemi H, Tromp G, et al. The Electronic Medical Records and Genomics (eMERGE) Network: past, present, and future. Genet Med 2013; 15: 761-771.

11. McCarty CA, Chisholm RL, Chute CG, et al.; eMERGE Team. The eMERGE Network: a consortium of biorepositories linked to electronic medical records data for conducting genomic studies. BMC Med Genomics 2011;4:13.

12. Lemke AA, Wu JT, Waudby C, Pulley J, Somkin CP, Trinidad SB. Community engagement in biobanking: Experiences from the eMERGE Network. Genomics Soc Policy 2010;6:35-52.

13. Kho AN, Pacheco JA, Peissig PL, et al. Electronic medical records for genetic research: results of the eMERGE consortium. Sci Trans/ Med 2011;3:79re1. 
14. Hartzler A, McCarty CA, Rasmussen LV, et al. Engaging stakeholders in informatics to integrate genomic information into the electronic health record (EHR): experiences from the eMERGE Network. Genet Med, 2013;15:792-801

15. Marsolo K, Spooner SA. Clinical genomics in the world of the electronic health record. Genet Med, 2013;15:786-791

16. Tarczy-Hornoch P, Amendola A, Aronson SJ, et al. A survey of informatics approaches to whole exome and whole genome clinical reporting in the electronic medical record. Genet Med, 2013;15: 824-832

17. Overby $\mathrm{CL}$, Kohane I, Williams MS, et al. Opportunities for genomic clinical decision support interventions. Genet Med, 2013;15:817-823
18. Kho AN, Rasmussen LV, Connolly JJ, et al. Practical challenges integrating genomic data into the electronic health record. Genet Med, 2013;15:772-778.

19. Ury A. Storing and interpreting genomic information in widely deployed electronic health records systems (EHRs). Genet Med, 2013;15:779-785.

20. Chute CG, Ullman-Cullere M, Wood G, Lin SM, He M, Pathak J. Some challenges of big data in translational research. Genet Med, 2013;15:802-809

21. Peterson JF, Bowton EA, Field JR, et al. Electronic health record design and implementation for pharmacogenomics: a local perspective. Genet Med, 2013;15:833-841

22. Hazin A, Brothers KB, Malin BA, et al. Ethical, legal and social implications of incorporating genomic information into electronic health records. Genet Med, 2013;15:810-816 